\title{
DISTRIBUTION OF FOOT AND MOUTH DISEASE VIRUS SEROTYPES IN CATTLE OF BANGLADESH
}

\author{
M.S. Islam, M.A. Habib, P.C. Saha, P.M. Das and M.A.H.N.A. Khan ${ }^{*}$ \\ Department of Pathology, Faculty of Veterinary Science, Bangladesh Agricultural University, \\ Mymensingh-2202, Bangladesh
}

\begin{abstract}
Foot and mouth disease (FMD) is a highly contagious viral infection in cloven hoofed domestic and wild animals and endemic in many countries of the world including Bangladesh. Clinical investigation was carried out to identify natural cases of FMD and characteristics signs of FMD like salivation, ulceration in oral and pedal tissues and lameness was seen. The specific serotypes of FMD viruses involved in infected cattle were, therefore, identified using reverse transcriptase polymerase chain reaction ( $R T-P C R)$. Samples $(N=97)$ from oral lesions was collected from infected cattle from seven divisions of Bangladesh during May to December, 2013. Viral RNA was extracted from the infected oral tissues and FMD virus specific uniplex RT-PCR was designed to detect FMD viruses. Multiplex RT-PCR was adapted to detect serotype specific amplicons. Out of 97 samples tested in uniplex and multiplex RT-PCR, 92 and 90 samples showed amplification reaction for FMD virus and viral serotypes respectively. Among the 90 FMD virus specific positive identification, single infectivity due to FMD viral Serotypes O, A and Asia 1 were seen in 56 (62.2\%), 13 (14.4\%) and 16 (17.8\%) cases respectively. Three cattle (3.3\%) were co-infected with FMD viral Serotypes $O$ and Asia 1 and two (2.2\%) with FMD viral Serotypes $O$ and A. FMD viral serotype $O$ was dominating all over the country followed by Asia1 and A. Cattle of Bangladesh were infected with FMD viral serotype $\mathrm{O}, \mathrm{A}$ and Asia 1 alone or in combination. The RT-PCR protocols designed and adapted successfully detected FMD viruses and viral serotypes in a fraction of the time required for virus isolation and serological detection. These RT-PCR protocols can be used for rapid serotyping of FMD viruses from filed infectivity and selection of vaccine viruses.
\end{abstract}

Keywords: Amplicon, FMD, FMDV, RNA, RT-PCR, serotype, vaccine

\footnotetext{
*Corresponding author email: hadi.khan@bau.edu.bd
}

Received: 08.12.2016 


\section{INTRODUCTION}

Foot and mouth disease (FMD) is an acute, highly contagious viral disease affecting all cloven-hoofed animals including cattle, swine, sheep, goats, wild pigs, wild ruminants and buffaloes. Clinically, FMD is characterized by vesicles formation in the mouth and on the muzzle, feet and sometimes on udders. Severe myocarditis causes high rate of mortality in infected newborns. The infection in adult animals is rarely lethal, it results in significant losses due to a decrease in production through reduced milk yield, loss of weight, abortions, delayed conception, and the restriction on international trade of animals and animal products (James and Rushton, 2002; Grubman and Baxt, 2004). Foot and Mouth disease virus (FMDV) is a nonenveloped, single stranded and positive sense RNA virus of the genus Aphthovirus belonging to the family Picornaviridae (Mumford, 2007). There are seven FMD viral serotypes namely O, A, C, SAT1, SAT2, SAT3, Asia1 and about 65 subtypes; vaccination with one serotype or subtype does not confer solid protection against others (Brown , 2003; OIE 2006; Saiz et al., 2002). Serotypes O and A are widely distributed, where as serotypes SAT1, SAT2 and SAT3 are restricted to Africa and serotype Asia1 to Asia (Knowles, and Samuel, 2003), rare incursions into other regions have been recorded. The last outbreak due to serotype C FMDV was reported in Bangladesh during 1996 (Kitching, 1998) and in Ethiopia during 2005 (WAHID 2009) and serotype $C$ viruses may no longer exist outside the laboratories.

FMD is endemic in Bangladesh (Islam et al., 2001) and sequence analysis of the capsid protein encoding genome region of the isolates collected in 1999 and 2000 showed that serotype $\mathrm{O}$ and serotype A were circulated in Bangladesh (Loth et al., 2011). FMD viruses isolated from outbreak areas in Bangladesh in late 2009 were also belonged to the viral serotype O (Nandi et al., 2013). Recently, the country has faced severe outbreaks of FMD due to serotypes $\mathrm{O}$ and $\mathrm{A}$, which is closely related to the viruses circulating in India and Nepal (Sarker et al., 2011). Diagnosis of FMD is commonly made by observing clinical signs. The viral serotypes in the laboratory can be done by virus isolation, demonstration of the FMD viral antigens or nucleic acid in a sample tissue or fluid by RT-PCR (OIE 2009). Detection of specific anti viral antibodies can also be made by using ELISA. This study was aimed to identify the naturally infected cattle by clinical examination and detect FMD viruses and viral serotypes from infected cases by means of RT-PCR, a sensitive, reliable and specific laboratory technology. Detection of FMD viral serotypes is required to understand distribution pattern of FMD, prepare vaccine seed using specific serotypes and design future preventive strategies.

\section{MATERIALS AND METHODS}

\section{Investigation of infected cattle and collection of samples}

The infected cattle were investigated for the presence of ulcerative lesions in oral and pedal tissues and salivation. The salivation was visible by distance 
observation and oral and pedal lesions were investigated before collecting samples. A total of 97 clinically ill cattle were examined and samples (epithelial tissues from oral lesions) were collected in transport media (MEM with antibiotic and antimycotic agents). Samples were collected (Figure 1) randomly from selected areas of Barisal $(n=9)$, Chittagong $(n=15)$, Dhaka $(n=25)$, Khulna $(n=10)$, Rajshahi $(n=16)$, Rangpur $(\mathrm{n}=11)$ and Sylhet $(\mathrm{n}=11)$ divisions of Bangladesh during May to December, 2013. The samples were shifted to the laboratory, Department of Pathology, Faculty of Veterinary Science, Bangladesh Agricultural University, Mymensingh in chilled condition and preserved at $-80^{\circ} \mathrm{C}$ until used in RNA extraction and RT-PCR detection of the viruses.

\section{RNA extraction and R-T PCR detection of viruses}

Total RNA was extracted from oral tissue homogenate using RNeasy Mini Kit (Qiagen \#74106) following manufacturer's instruction. The concentration and purity of extracted RNA were measured by analyzing the samples in a spectrophotometer (Islam et al., 2016). FMD virus specific uniplex RT-PCR (uRT-PCR) was carried out with 50-60ng RNA/ reaction to detect fragment of Lpro gene of FMD viruses with designed primer pairs FMDF (5'-ctctctgttacacgctctcag-3') and FMDR (5'cgaacacagcgtgttcttgcc-3'). The SuperScript III one step RT-PCR kit with Platinum Taq (Invitrogen, USA) was used to amplify Lpro gene. Extracted RNA of the uRTPCR positive samples was then subjected to multiplex RT-PCR (mRT-PCR) for the detection of viral serotypes using serotype specific published primers (Table 1). The RT-PCR was carried out with $25 \mu 1$ reaction volume (Table 2 ) in a thermocycler using the thermal profile stated in table 3 . Finally, the cDNAs were gel electrophoresed in a transilluminator (Alphaimager HP, California, USA) and the serotypes of the viruses were identified by observing selecting bands in agarose gel.

Table 1. Primer sequences used for the detection of FMD viral serotypes by using multiplex RT-PCR (Callens and De Clercq, 1997)

\begin{tabular}{l|ll|ll}
\hline $\begin{array}{l}\text { Primer } \\
\text { Name }\end{array}$ & Sequence (5'-3') & Orientation & $\begin{array}{l}\text { Amplicon } \\
\text { size(bp) }\end{array}$ & Serotypes \\
\hline P33 & AGCTTGTACCAGGGTTTGGC & Reverse & & \\
P38 & GCTGCCTACCTCCTTCAA & Forward & 402 & O \\
P40 & GTTTCTGCACTTGACAACACA & Forward & 596 & C \\
P74 & GACACCACTCAGGACCGCCG & Forward & & \multirow{2}{*}{ Asia1 } \\
P75 & GACACCACCCAGGACCGCCG & Forward & 292 & \\
P76 & GACACCACACAAGACCGCCG & Forward & & \\
P77 & GACACGACTCAGAACCGCCG & Forward & & A \\
P110 & GT(G:A:T:C)ATTGACCT(G:A:T:C) & Forward & 732 & \\
\hline
\end{tabular}




\section{Distribution analysis of viral serotypes}

Results obtained from the RT-PCR with the samples collected from different divisions of Bangladesh was analyzed by using Microsoft Office excel 2007. The percentage analysis was carried out using Office excel and fraction point 0.45 and above was considered as single point. Distribution of FMD viral serotypes O, Asia 1 and A throughout the country was plotted.

Table 2. Composition of reaction mixture for $\mathrm{mRT}-\mathrm{PCR}$. The amplification reaction was carried out in $25 \mu \mathrm{l}$ volume

\begin{tabular}{l|l|l}
\hline SL. No. & Components & Volume \\
\hline 1 & 2X reaction mixture & $12.5 \mu 1$ \\
2 & Forward primer $(20 \mathrm{pmol} / \mu \mathrm{l})-\mathrm{P} 38$ & $0.5 \mu 1$ \\
3 & Forward primer $(20 \mathrm{pmol} / \mu \mathrm{l})-\mathrm{P} 40$ & $0.5 \mu 1$ \\
4 & Forward primer $(20 \mathrm{pmol} / \mu \mathrm{l})-\mathrm{P} 74$ & $0.5 \mu 1$ \\
5 & Forward primer $(20 \mathrm{pmol} / \mu \mathrm{l})-\mathrm{P} 75$ & $0.5 \mu 1$ \\
6 & Forward primer $(20 \mathrm{pmol} / \mu \mathrm{l})-\mathrm{P} 76$ & $0.5 \mu 1$ \\
7 & Forward primer $(20 \mathrm{pmol} / \mu \mathrm{l})-\mathrm{P} 77$ & $0.5 \mu 1$ \\
8 & Forward primer $(20 \mathrm{pmol} / \mu \mathrm{l})-\mathrm{P} 110$ & $0.5 \mu 1$ \\
9 & Reverse primer $(20 \mathrm{pmol} / \mu \mathrm{l})-\mathrm{P} 33$ & $0.5 \mu 1$ \\
10 & Taq polymerase enzyme & $0.5 \mu 1$ \\
11 & RNAse out & $1 \mu 1$ \\
12 & Nuclease free water & $2 \mu 1$ \\
13 & RNA template & $5 \mu 1$ \\
Total volume & $25 \mu 1$ \\
\hline
\end{tabular}

\section{RESULTS AND DISCUSSION}

Foot and Mouth Disease (FMD), popularly known as Apthus fever, khura, jhora appeared as an acute illness in cattle. FMD is a highly contagious viral disease of almost all the cloven-hoofed domestic animals including cattle, buffalo, sheep, goats and swine (Ullah et al., 2015). Infected animals showed high fever, vesicular lesions and ulceration in oral and pedal skin (Figure 1). The ulcerative lesions in oral mucosa and interdigital skin was used to establish a diagnosis of FMD (OIE, 2009). In this study the vesicular lesion were not seen in any case and the epithelial tissues from the infected oral lesions were, therefore, collected to extract viral RNA and detection of Lpro and VP1 genes of FMD viruses in RT-PCR. 


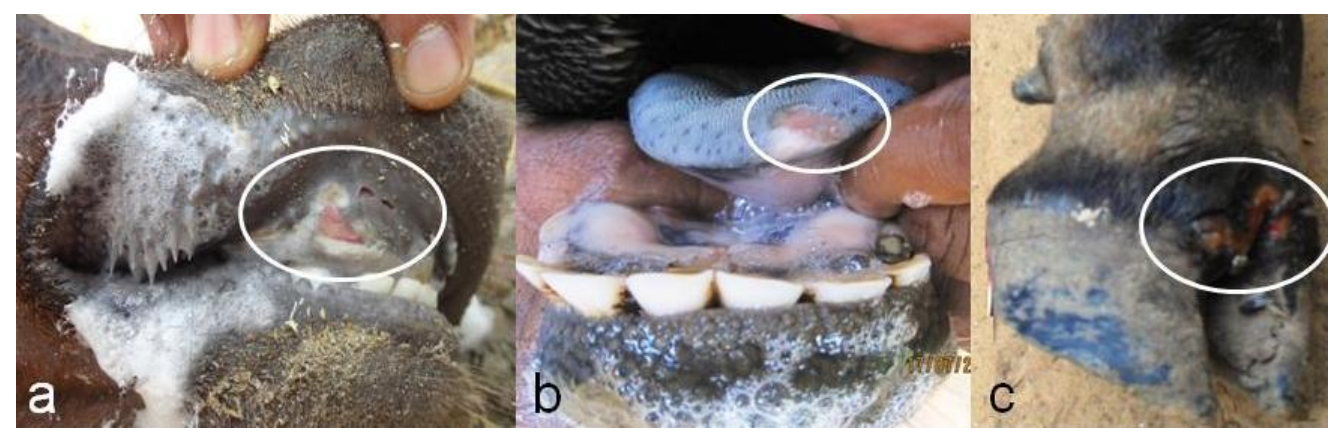

Figure 1. Naturally infected cattle due to FMD. Profuse frothy salivation (a and b), ulceration and erosion in oral and pedal tissues were commonly seen.

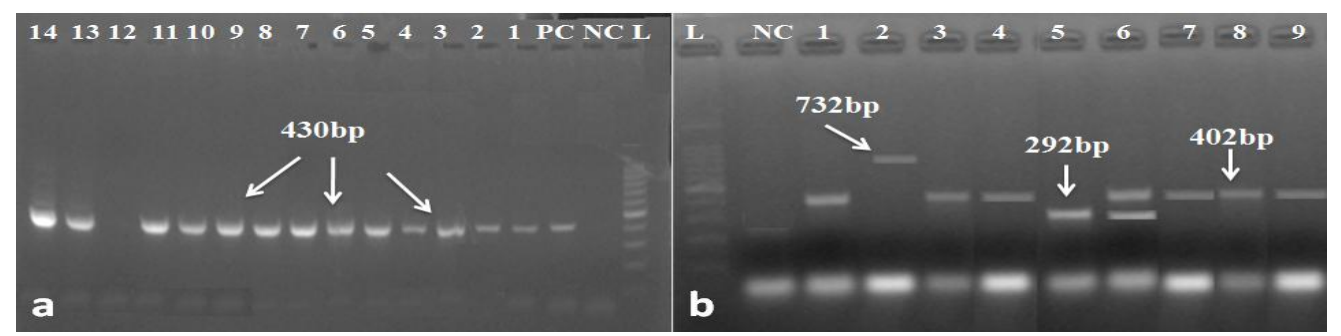

Figure 2. Viral RNA (L-pro gene) from the field samples (a) were tested in uRT-PCR detection of FMD viruses and in positive cases 430bp amplicon was generated. The lane $\mathrm{L}$ is for 100bp Ladder, NC is for negative control, PC is for positive control and lane 1 to 14 and 1 to 9 are for test samples. Viral RNAs (b) was also used in mRT-PCR detection of FMD viral serotypes and generation of 292bp, 402bp and 732bp amplicons were specific for FMD viral Serotype Asia1, O and A respectively. Mixed infection was detected in RT-PCR (b, lane 6).

FMD in cattle from naturally infected cattle was diagnosed by observing characteristics clinical signs. But most difficulty is lying with the detection of viral serotypes. FMD viruses and viral serotypes were identified from field samples using uniplex and multiplex RT-PCR. Results of uRT-PCR (Figure 2a) showed amplification of $430 \mathrm{bp}$ amplicons in 92 cases as documented in agarose gel; the amplification was specific for FMD viruses (Islam et al., 2016). A mRT-PCR was standardized using published primer sequences (Callens and De Clercq, 1997). The reverse primers of this RT-PCR was designed from a sequence of VP1 gene common to all serotypes and forward primers was designed from selective region of VP1 gene; that determine the specific serotype of FMD. The extracted RNA from the field samples $(\mathrm{N}=92)$ while tested in mRT-PCR, 292bp, 402bp and 763bp amplicons (Figure 2b) were generated in 90 cases; there were specific for FMD viral serotypes Asia1, $\mathrm{O}$ and A respectively. FMD viral Serotype O, A, Asia1 were detected alone in $56(62 \%), 13(14 \%)$ and $16(18 \%)$ cases respectively (Table 3). Three cattle (3\%) were co-infected with FMD viral Serotypes O and Asia 1 and two $(2 \%)$ with FMD 
viral Serotypes O and A (Table 3). There was lacking of non-specific amplification in RT-PCR. Specificity of the RT-PCR was found higher and detected FMD viral serotypes O, A and Asia 1 in a reaction (Reid et al., 2000). The multiplex RT-PCR did not generate 596bp amplicon and FMD viral serotype $C$ was, therefore, remain undetected in this study.

\section{Geographic distribution of FMD viral serotypes in Bangladesh}

Results of RT-PCR showed that there was about $62 \%, 13 \%$ and $18 \%$ infectivity in cattle due to FMD viral serotypes $\mathrm{O}, \mathrm{A}$ and Asia1. About $3 \%$ and $2 \%$ co-infectivity in cattle was seen due to FMD viral serotype $\mathrm{O}$ and Asia1 and $\mathrm{O}$ and $\mathrm{A}$ respectively (Table 4). FMD viral serotype $\mathrm{O}$ found dominating in cattle followed by serotype Asia1 and A (Figure 3). FMD viral serotype $\mathrm{O}$ was dominating and common in all division. FMD viral serotype $\mathrm{O}$ and Asia 1 was prevailing at an equal level at Rajshahi division of Bangladesh. Serotype A was not detected in Chittagong, Khulna and Sylhet divisions where as serotype Asia1 was absent in Barisal division (Figure 4 \& Table 3).

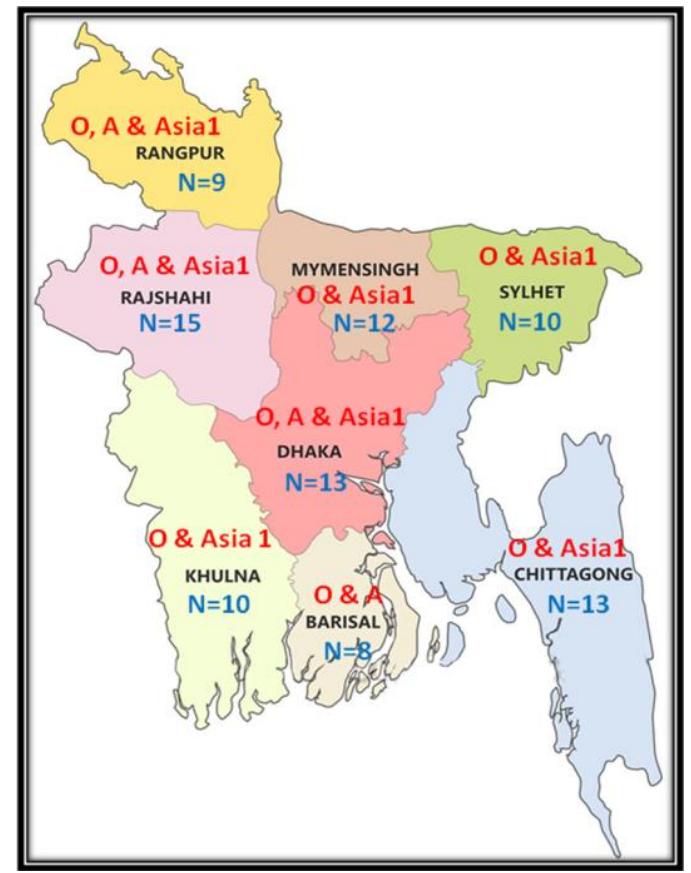

Figure 3. Map of Bangladesh showing different divisions and distribution FMD viral serotypes. $\mathrm{N}$ represent the number of cases examined in this study.

Previously the dominancy of FMD viral serotype $\mathrm{O}$ in cattle was also reported in Bangladesh during the year 2011 (Sarker et al., 2011) and identified 80\%, 12\% and $8 \%$ infectivity due to FMD viral serotype O, Asia1 and A in cattle respectively. The 
dominancy of FMD viral serotype $\mathrm{O}$ in 2011 was much higher than the present infectivity. Recently a different observation was noted in Bangladesh and found dominancy of FMD viral serotype A over Asia1 (Hossen et al., 2014); this might be due to differences in the coverage of sample collection areas. Infectivity of cattle due to FMD viral serotypes O (80\%), A (8\%) and Asia1 (12\%) in India was also reported during April 2006 to March 2011 (Subramaniam et al., 2013), where FMD viral serotype $\mathrm{O}$ was dominating. FMD viral serotypes $\mathrm{O}$, A and Asia1 are consistently circulating in Bangladesh, Bhutan, India, Nepal, and Sri Lanka (Pool 2) with a dominancy of serotype O (FAO, 2015). There is unlimited and uncontrolled animal movements throughout the SAARC countries, the scenario is worsening during religious festival. Now a day cross border cattle movements through Bangladesh, India and Myanmar although reduced but there are still some gate/point that allows unlawfully movement of cattle. Cattle, buffaloes, sheep and goats movement throughout the cross border and in country make ubiquitous distribution of FMD viral serotypes O, Asia1 and A in the SAARC countries (Siddique et al., 2014; Subramaniam et al., 2015). The FMD viral serotype $O$ has been dominating in the SAARC countries. From the very beginning the FMD viral serotypes $O$ was dominated over all other serotypes (Chowdhury et al., 1993; Pervin et al., 2011; Ullah et al., 2015) and this situation still exists (Islam et al., 2015). In country movement of animals may have contributed spreading of FMD and viral serotype.

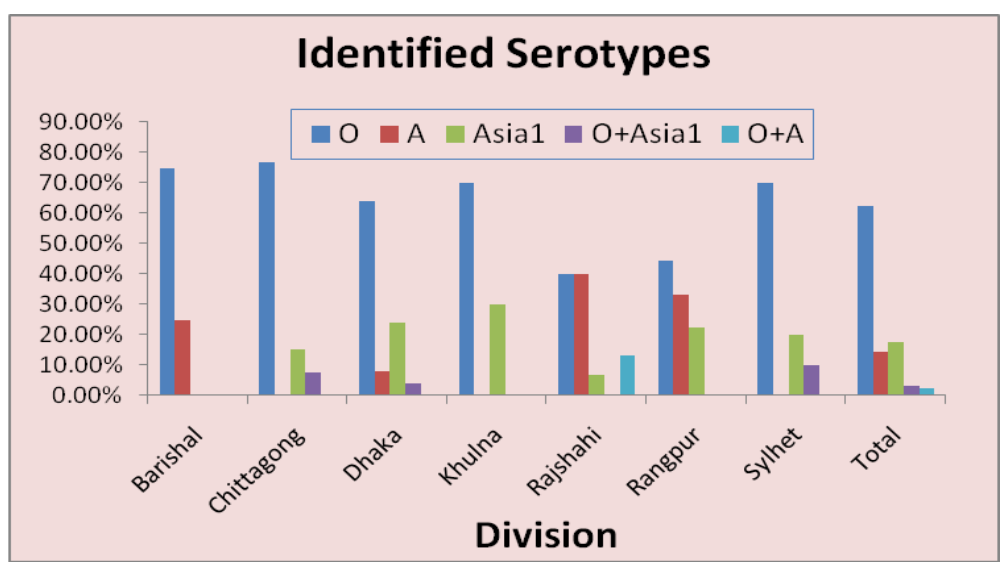

Figure 4. Division wise distribution of FMD viral serotypes in Bangladesh. Mixed infection due to FMD viral serotypes O plus Asia1 and O plus A was also cases 
Table 3. Results of uniplex and multiplex RT-PCR to detect FMD viruses and viral serotypes From the natural outbreaks in cattle

\begin{tabular}{|c|c|c|c|c|c|c|c|c|}
\hline \multirow[t]{2}{*}{ Division } & \multirow{2}{*}{$\begin{array}{c}\text { Number } \\
\text { of } \\
\text { samples }\end{array}$} & \multirow{2}{*}{$\begin{array}{l}\text { Positive in } \\
\text { uRT-PCR } \\
(\%)\end{array}$} & \multirow{2}{*}{$\begin{array}{c}\text { Positive in } \\
\text { mRT-PCR } \\
(\%)\end{array}$} & \multicolumn{5}{|c|}{ FMDV Serotypes identified by mRT-PCR (\%) } \\
\hline & & & & $\mathrm{O}$ & A & Asia1 & $\mathrm{O}+$ Asia1 & $\mathrm{O}+\mathrm{A}$ \\
\hline Barishal & 9 & $8(88.89)$ & $8(100)$ & $6(75)$ & $2(25)$ & - & - & - \\
\hline Chittagong & 15 & $14(93.33)$ & $13(92.85)$ & $10(76.92)$ & - & $2(15.38)$ & $1(7.69)$ & - \\
\hline Dhaka & 25 & $25(100)$ & $25(100)$ & $16(64)$ & $2(8)$ & $6(24)$ & $1(4)$ & - \\
\hline Khulna & 10 & $10(100)$ & $10(100)$ & $7(70)$ & - & $3(30)$ & - & - \\
\hline Rajshahi & 16 & $15(93.75)$ & $15(100)$ & $6(40)$ & $6(40)$ & $1(6.67)$ & - & $2(13.33)$ \\
\hline Rangpur & 11 & $10(90.91)$ & $9(90)$ & $4(44.44)$ & $3(33.33)$ & $2(22.22)$ & - & - \\
\hline Sylhet & 11 & $10(90.91)$ & $10(100)$ & $7(70)$ & - & $2(20)$ & $1(10)$ & - \\
\hline Total & 97 & $92(94.85)$ & $90(97.83)$ & $56(62.22)$ & $13(14.44)$ & $16(17.78)$ & $3(\mathbf{3 . 3 3})$ & $2(2.22)$ \\
\hline
\end{tabular}

\section{CONCLUSIONS}

This study designed and adapted uniplex and multiplex RT-PCR protocols for rapid detection of FMD viruses and serotyping of the viruses using RNA extracted from the infected oral tissues. Out of 97 bovine samples tested in uniplex RT-PCR, 430bp amplicon was generated in 92 cases (94.85\% detection level), suggestive for infectivity due to FMD viruses. While viral RNA from the URT-PCR test positive samples $(\mathrm{N}=92)$ were used in $\mathrm{mRT}$-PCR protocol, specific FMD viral serotypes was detected in 90 cases (98\% test positivity). The RT-PCR protocols appeared highly sensitive (more than 90\%) in terms of detecting FMD viruses and viral serotypes and were specific. FMD viral serotypes $\mathrm{O}$, Asia1 and A were circulated in cattle during 2013 outbreaks in Bangladesh. FMD viral serotype $O$ found dominating over serotype Asia 1 and A. Mixed infection with $\mathrm{O} \&$ Asia1 and $\mathrm{O}$ and $\mathrm{A}$ was also reported. Infectivity in cattle due to FMD viral serotype $\mathrm{C}$ is still absent in Bangladesh. Overlapping of infectivity due to FMD viral serotypes as seen in this study recommend the use of polyvalent vaccine consisting of FMD viral serotypes $\mathrm{O}$, $A$ and Asia 1. The FMD viral vaccines used in Bangladesh lack information about their antigenic and genetic properties and protective efficacy; these may contribute vaccination failure and regular outbreak of FMD in the field.

\section{ACKNOWLEDGEMENTS}

Thanks are due to the Krishi Gobeshona Foundation (KGF), Farmgate, Dhaka, Bangladesh for funding the research. Sincere appreciation is also extended to the Department of Pathology, Faculty of Veterinary Science, Bangladesh Agricultural University, Mymensingh-2202, Bangladesh for providing internal resources and laboratory facilities to complete the research. 


\section{REFERENCES}

Brown, F. 2003. The history of research in foot and mouth disease. Virus Research, 91(1):3-7

Chowdhury, S.M.Z.H., Rahman, M.F., Rahman, M.B. and Rahman, M.M. 1993. Strains of foot and mouth disease virus in different districts of Bangladesh. Asian-Australian Journal of Animal Sciences, 9: 315-317

FAO, 2015. Food and Agriculture Organization of the United Nations, Foot-and-Mouth Disease Situation, Monthly Report, November, 2015

Grubman, M.J. and Baxt, B. 2004. Foot-and-mouth disease. Clinical Microbiology Review, 17(2): 465-493

Hossen, M.L., Ahmed, S., Khan, M.F.R., Rahman, M.T., Saha, S., Nazir, K.H.M.N.H., Rahman, M., Islam, M.A. and Rahman, M.B. 2014. Typing of Foot and Mouth Disease Virus Circulating in Bangladesh by Reverse Transcription Polymerase Chain Reaction. Journal of Veterinary Advances, 4(12):778-785

Islam, M.S., Ruba, T., Habib, M.A., Rima, U.K., Hossain, M.Z., Saha, P.C., Das, P.M. and Khan, M.A.H.N.A. 2016. Sequencing and Translational Analysis Revealed Huge Mutation in the N-Terminus End of Leader Proteinase (Lpro) Gene of Foot and Mouth Disease Viruses Isolated From Cattle in Bangladesh. IOSR Journal of Agriculture and Veterinary Science (IOSR-JAVS), 9(2): 24-30

Islam, M.A., Rahman, M.M., Adam, K.H. and Marquardt, O. 2001. Epidemiological implications of the molecular characterization of foot-and-mouth disease virus isolated between 1996 and 2000 in Bangladesh. Virus Genes, 23: 203-210

James, A. D. and Rushton, J. 2002. The economic s of foot-and-mouth disease. Revue Scientifique-et-Technique, Office International des Epizooties, 21: 637-644

Kitching, R.P. 1998. A recent history of foot and mouth disease. Journal of Comparative Pathology, 118: 89-108

Knowles, N.J. and Samuel, A.R. 2003. Molecular epidemiology of foot and mouth disease virus. Virus Research, 91: 65-80

Loth, L., Osmani, M.G., Kalam, M. A., Chakraborty, R.K., Wadsworth, J., Knowles, N.J., Hammond, J.M. and Benigno, C. 2011. Molecular Characterization of Foot-and-Mouth Disease Virus: Implications for Disease Control in Bangladesh. Transboundary and Emerging Diseases, 58: 240-246

Mumford, J.A. 2007. Vaccines and viral antigenic diversity. Revue scientifique et technique, 26: 69-90

Nandi, S.P., Rahman, M.Z., Momtaz, S., Sultana, M. and Hossain, M.A. 2013. Emergence and Distribution of Foot-and-Mouth Disease Virus Serotype A and O in Bangladesh. Transboundary and Emerging Diseases, Short Communication 1-4

OIE, 2009. Principles of veterinary vaccine production. In: Manual of diagnostic tests and vaccines for terrestrial animals. Version adopted May (2006). Chapter 1.1.7

Pervin, M., Faruque, M.H., Khanm, S.S., Chowdhury, E.H. and Khan, M.A.H.N.A. 2011. Reverse transcription polymerase chain reaction (RT-PCR) for the detection and typing of foot and mouth disease virus in naturally infected cattle of Bangladesh. Bangladesh Journal of Progressive Science and Technology, IX(I): 009-012 
Reid, S.M., Ferris, N.P., Hutchings, G.H., Samuel, A.R.and Knowles, N.J. 2000. Primary diagnosis of foot-and-mouth disease by reverse transcription polymerase chain reaction. Journal of Virological Methods, 89(1-2):167-76

Saiz, M., Nunez, J.I., Jimenez-Clavero, M.A., Baranowski, E. and Sobrino, F. 2002. Foot and Mouth disease virus: biology and prospects for disease control. Microbes and Infection, 4: 1183-1192

Sarker, S., Talukder, S., Haque, M.H., Islam, M.H. and Gupta, S.D. 2011. Epidemiological study on foot-and-mouth disease in cattle: Prevalence and risk factors assessment in Rajshahi, Bangladesh. Wayamba Journal of Animal Science, 3: 71-73.

Siddique, M.A., Ullah, H., Nandi, S.P., Chakma, D., Sultana, M. and Hossain, M.A. 2014. Molecular Characterization of Foot-and-Mouth Disease Virus Type O from Wild Pig in Bangladesh. Bangladesh Journal of Microbiology, 32(1-2): 41-45

Subramaniam, S., Mohapatra, J.K., Das, B., Sharma, G.K., Biswal, J.K., Mahajan, S., Misri, J., Dash, B.B. and Pattnaik, B. 2015. Capsid coding region diversity of re-emerging lineage $\mathrm{C}$ foot-and-mouth disease virus serotype Asia1 from India. Archives of Virology, 160(7): 1751-1759

WAHID, 2009.OIE World Animal Health Database. Available at: http://www.oie.int/wahis/public.php? page=country_status \& year=2009 (accessed February 14, 2010) 